\title{
An Experiment: Hiring Discrimination on Appearance, Especially on Women
}

\author{
Xinran Gao* \\ NCPA-GuangZhou Fok Ying Tung High School, Shenzhen, 518000, China, 2230089@ncpachina.org
}

\begin{abstract}
It's a common phenomenon that appearance discrimination occurring in Hiring condition, however, in China, things are a little different. Compare to other countries' gender gap decreased gradually, the difference of gender in China kept rising. An experiment was built up in this article, both implicit and explicit bias were measured and discussed: made-up resumes were sending to hiring managers and callback rates were recorded, then questionnaires were sending to hiring managers for IAT test result as well. The both result were been discussed and possible future plan were proposed on appearance discrimination when it comes to hiring condition, especially, when it comes to women.
\end{abstract}

Keywords: Appearance discrimination, obese females, gender gap.

\section{INTRODUCTION}

Discriminations could occur from varieties aspects, it's so easy to have stereotypes/prejudice on people who have different colour, who come from different nation, different gender, or even could just because of people is late for working. It's quite shocking that stereotypes could vary people's performance in such a way. Appearance anxiety has already become a common phenomenon not only in China but worldwide: Korean/German women had reported higher level of body shame and appearance anxiety, in addition, body dissatisfaction and eating disorders were always relevant and may cause health problems [1]. What's public's attitude about people's appearance? Based on an observational study's poll results in China $(\mathrm{n}=17,702), 75 \%$ young people (under the age of 33) believed being more attractive would bring them more or less competitiveness in the society, what's more, $51.3 \%$ of them responded that they have considered doing facelift some day [2].

The situation is, men's body fate percentage are much lower than women's, men with larger weight could be acknowledged lighter than women when they actually are in the similar weight, therefore, the problem occurs, the requirements on women's weight are stricter than men would be asked for. Adult obesity rate stands $42.4 \%$ in US, which had increased by $26 \%$ since 2008 , and this was the very first time the national rate had passed $40 \%$ [3]. Social-economic factors could give rise to this result, such as poverty and discriminations, which was a thought-provoking causation. Would hiring discrimination include obesity and end up with unfair treatment, which unfortunately contribute to citizens' poverty, indirectly cause an endless circle? The existing literatures mostly focus on western countries, and very few studies put attention to the difference that caused by gender, therefore, we turned our eyes on an Asian country, China, that we've already known that China had surpassed become the most obese people in the wold in recent years, for further study.

More than $50 \%$ adults in China had classified overweight, in particular, $16.4 \%$ are obese, in 2020 [4]. Based on the past studies, there are a lot of stereotypes happened on overweight and obese people, generally, less active, less productive, less successful, etc, which result in less recommendations for hiring than others [5]. Here comes to our concern, in such a huge statistical data record, will these people encounter more hiring discriminations than others even though there would not be face-to-face interviews for hiring managers to directly interact with them? We believe that overweight/obese people will experience more or less discriminations due to hiring managers' automatically stereotypes; secondly, we also deem that women in the same weight situation would experience more discriminations than men do. Based on our hypothesis, we have designed a field study and evaluated the result based on the callback rates. IAT(Implicit Associations Test) is being used in our study in order to predict hiring managers' reacting behaviours. 


\section{STUDY DESIGN}

We created 18 resumes in total, 9 for each gender $(n=18)$. Basically, the resumes included all the applicants' names, email addresses, phone numbers, objectives, skills, education backgrounds, etc (Fig 1 for example). Very importantly, the profiles, which were used in order to show hiring managers the different types of weight (normal/overweight and obesity) among applicants. Lots of occupations such as receptionist and waitress, are naturally having requirements on workers' appearances, which were been excluded in our first decision. Sales assistant and accountant's occupations were being chosen for our subjects, since hiring managers in these two positions will mostly focused on people's professional knowledge rather than pay attention to more attractive appearance.

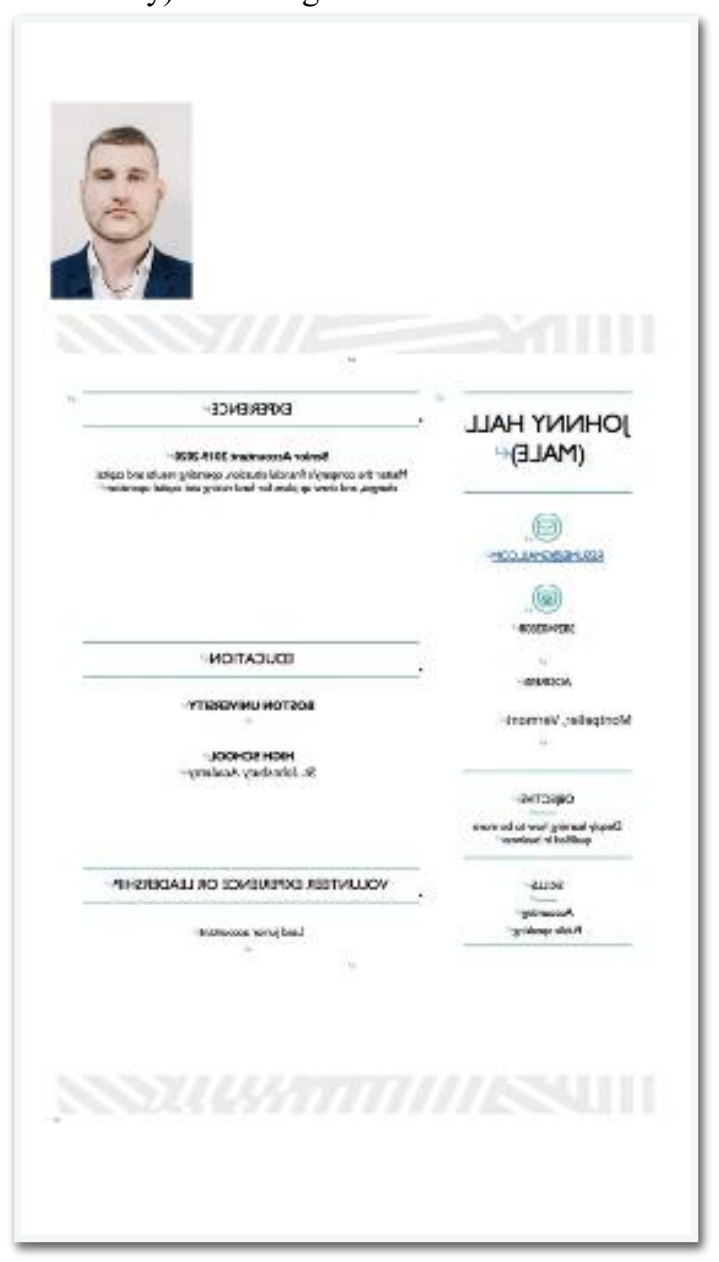

Fig 1 Applicants Resume

The made-up applicants had very similar information in every resumes, such as academic qualification, relevant work experience, and being educated in almost the same type of universities (ranked similarly). We controlled the variables to ensure that the info was mostly the same, hence minimising possible bias happening.

Six companies would be randomly chosen for our study, participants from companies (hiring managers) would receive the resumes. Afterwards, we chose six photos from internet that were created (three women, three men), then photoshopped them into three types: normal weight, overweight and obesity.
Set normal weight as 1, overweight as 2 and obese as 3. There are woman A, B and C, man D, E and F (Fig 2 Study Design). Randomly combined them, for example, combined A1, B2 and C3 as a group (for women), which included all types of weight that we are going to test about; then using the same principle, combined D1, E2 and F3 (for men). Distributed both gender into 3 groups (3 different types of weight for each gender as a group), then sent out resumes in group to companies twice, once for sales assistant occupation, and once for accountant. (3 companies are looking for sales assistant, 3 for accountant). 


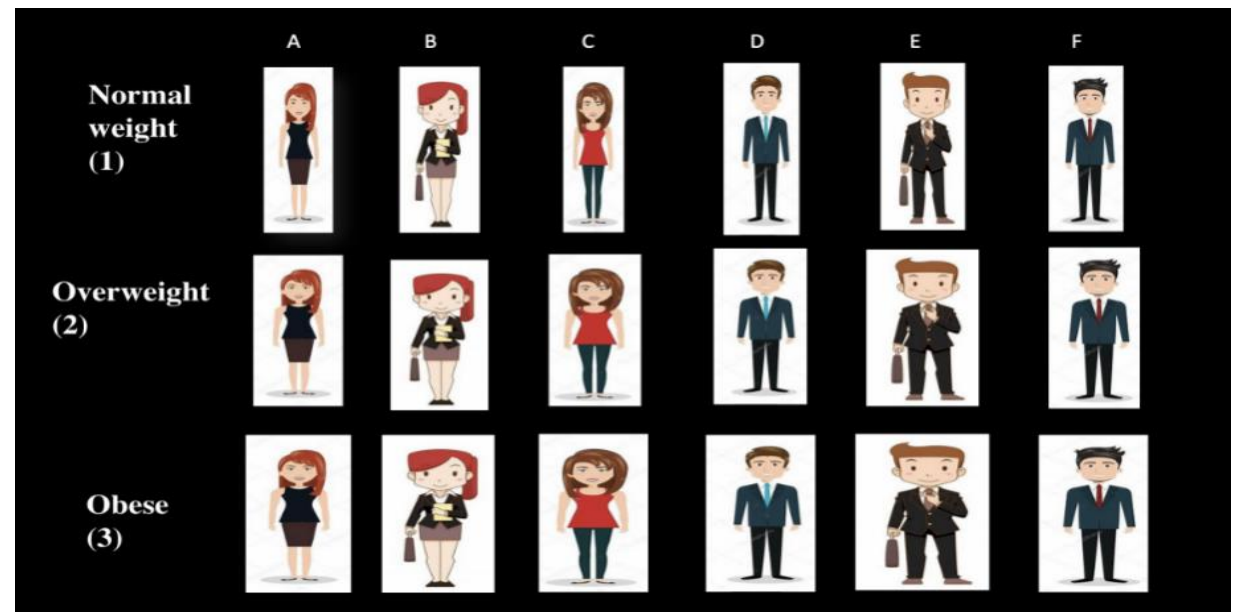

Fig 2 Study Design

\subsection{Measure}

Discriminatory behaviour would be measured by callback rates and the result of IAT test (we will sent to the hiring managers). Questionnaires were sent to participants for IAT testing, for instance, how do you think about obesity, do you have any family member is overweight, how do you think about gender equality, and so on, asking some specific questions to indicate gender/obesity discriminations.

We believe that IAT test will match our expected result, hiring manager who gets lower score on IAT test, will have high probability show more discrimination during the study, which means he/she would hire more people with normal weight. Furthermore, IAT test could tell us implicit bias that may not be classified by only doing the field study, we will understand more clearly whether the hiring managers holding gender discriminations or not.

After the experiment, we would inform hiring managers the purpose of the study, in case they really opposed and was unwittingly forced to participate in the experiment, they will be rewarded because of their contributions to the study. The result will be analysed, and then discussed about whether obesity/gender discrimination do exist because of people's stereotypes or not, and there are actually any disadvantages impacted on them.

\subsection{Result}

According to former studies on gender discriminations and the ratio of getting hired, in Chinese

market, gender gap had risen from $9.4 \%$ in 1990 to $14.1 \%$ in 2020 [6]. We could find out that gender gap (Fig 3 gender gap) is decreasing gradually in other countries [7], whereas the condition in China is getting more and more serious: before getting employed, women are easily being considered they need to contribute their time in taking care of family and children. Even if women have the same capacity as men do, they still get lower chance to be hired. We reasonably predicted the probability that normal weight males could be hired is $43 \%$, nearly $1 / 2$ chance. However, even though females are fighting for theirs rights actively, situation is still very serious, $27 \%$, the probability is half lower than men own. Overweight/obesity people, no matter females or males, their opportunities are much less than normal-weight individuals, and obese females who have chance to get an interview, is only $9 \%$. 


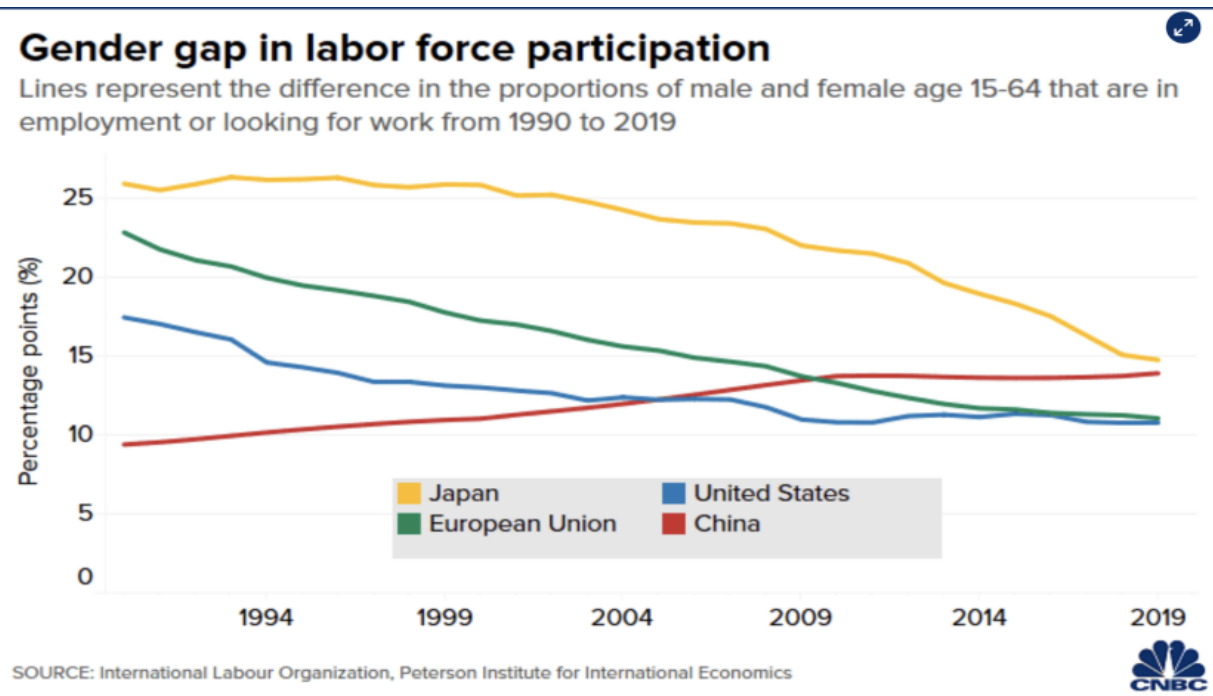

Fig 3 Gender Gap

As the result shows us (Fig 4 the expected result), people in normal weight have much more chance

to be employed, what's more, fat males get similar chance to be hired as normal weight females do. When people in different gender are in the same weight, males always have more opportunity to be hired than women do. According to what we saw in the gender gap (Fig 3 gender gap), this condition will be even more severe in China's job market, which may cause many problems happening in the society. For example, even though females have occupied $50 \%$ of the world's population, the rate of wealth owning for them is only $1 \%$. Also, in some places, women are still hardly to earn money, or to inherit property due to lack of rights [8] .

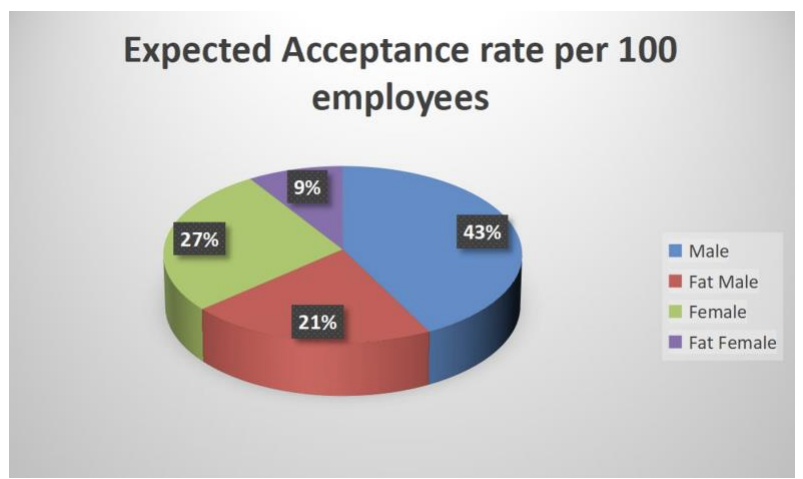

Fig 4 Expected Result

\section{CONCLUSION}

People always see obese people as those with less capacity in finishing daily tasks. Such discrimination would result in less salaries for these populations at start, and they might be regarded as groups with low competence. With low socioeconomic status, coworkers always treat them contemptuously since they are being questioned about leadership potential. The result is obvious, and is also being proved in our study: they will hardly get chance to demonstrate their abilities since most of them even could not get an interview, not to mention gaining the jobs with their talents.

Social trend could cause discriminations occurring: for a surprisingly funding, weight discrimination is even more serious than racial discrimination. For instance, in an experiment $(n=2,290)$ [9], almost $60 \%$ participants had reported hiring discriminations occurred at least once, $3 \%$ of overweight, $6 \%$ of obese and $28 \%$ of very obese. Again (in this data also), women had experienced much more discriminations than men had. The percentage respectively were $10 \%, 20 \%$ and $45 \%$; almost exceed half of the participants.

Overweight/obese people unfortunately get paid less than those with normal weight, by doing a statistical analysis, $93 \%$ of hiring managers are more willing to hire someone that is in normal weight, rather than hiring people who are visibly obese. Sadly, the market is even more hostile to females. Heavy women generally earned 9,000 dollars less on average, more heavier a woman is, she will get less and less paid in total [10].

Discrimination against obese women more than against obese men leads to more potential discrimination. Nevertheless, some researches have shown another discrimination direction, that is, underweight. Surprisingly, higher probability for men to be unemployed if they are underweight, which is a very different condition compare to overweight. Some overweight/obese people may choose to control their diet in an unhealthy approach, and eventually develop eating disorder, making the condition even worse than before. For example, anorexia nervosa and avoidant-restrictive food intake disorder, which are relevant to underweight possibility. In another case, bulimia disorder and bingeeating disorder will make people being more obese, and bringing about healthy problems in the end [11].

In European countries and America, they got laws about anti-discrimination to restrain excessive 
discriminations happening. For example, under the EPA (Equal Pay Act) in 1963, companies were prohibited to pay women less than man at work, some laws also restrained discriminations about sex orientation and tattoos, and so on [12]. However, in China, even though we do have laws talked about gender discrimination, but we do not have a very accurate regulations to standard obesity discrimination. That's something for the future plan.

\section{REFERENCES}

[1] Freiburg. i. Br.(Germany), 2010. The Role of Body Shame, Social Appearance Anxiety, and Body Checking Behaviour on Body Dissatisfaction and Disordered Eating Behaviours: A Cross-Cultural Study in Germany and Korea. https://www.freidok.unifreiburg.de/fedora/objects/freidok:7926/datastream s/FILE1/content

[2] Mo Hong'e(China), 2013. Young Chinese place high importance on looks: survey. http://www.ecns.cn/2013/08-07/76344.shtml

[3] Rhea Farberman(America),2020. The State of Obesity 2020: Better Policies for a Healthier America. https://www.tfah.org/report-details/stateof-obesity2020/\#: :text=The\%20U.S.\%20adult\%20obesity\% 20rate $\% 20$ stands $\% 20$ at $\% 2042.4 \% 20$ percent, $\% 20$ th e,by\%2026\%20percent $\% 20$ since $\% 202008$

[4] BBC News(UK),2020. Over half of Chinese adults overweight, study finds. https://www.bbc.com/news/world-asia-china55428530

[5] Karol V.Mason(University of Michigan), 1982. Employment of Discrimination Against the Overweight. https://www.bbc.com/news/worldasia-china-55428530

[6] Eva Zhang and Tianlei Huang(PIIE), 2020. Gender discrimination at work is dragging China's growth. https://www.bbc.com/news/world-asia-china55428530

[7] Yen Nee Lee(CNBC), 2020. Women in China are losing out in the workforce - that's bad news for the economy. https://www.bbc.com/news/world-asiachina-55428530

[8] Medical Ambassadors(CHE), 2021. Gender Inequality \& Community Health Evangelism. https://www.bbc.com/news/world-asia-china55428530

[9] Rebecca Puhl, $\mathrm{PhD}(\mathrm{OAC})$. Weight Discrimination: A Socially Acceptable Injustice. https://www.bbc.com/news/world-asia-china55428530

[10] EKU. Overweight and Underpaid: Weight Discrimination at Work https://safetymanagement.eku.edu/blog/overweight -and-underpaid-weight-discrimination-at-work/

[11] Brain \& Behaviours Research Foundation. Eating Disorders

FAQs. https://safetymanagement.eku.edu/blog/overweight -and-underpaid-weight-discrimination-at-work/

[12] Indeed for Employers. Employment Discrimination Laws: Three Things to Avoid. https://www.indeed.com/hire/c/info/employeediscriminationlaws?aceid=\&gclid=Cj0KCQjw4ImEBhDFARIsA GOTMj03OwZci11zrU5rY539qmIFwfzaMuNldYTATJPf OEYgOgA9wCqqRsaAo5DEALw_wcB 